\title{
Heterogeneity in breast cancer and the problem of relevance of findings
}

\author{
M. Aubele ${ }^{\mathrm{a}, *}$ and M. Werner ${ }^{\mathrm{b}}$ \\ ${ }^{a}$ GSF - National Research Center for Environment \\ and Health, Institute of Pathology, Neuherberg, \\ Germany \\ ${ }^{\mathrm{b}}$ Technische Universität München, Klinikum rechts \\ der Isar, Institute of Pathology, Munich, Germany
}

Received 15 August 1999

Accepted 16 November 1999

Many attempts are made to identify critical genetic events responsible for the development and progression of breast cancer. There is increasing evidence that breast cancer is a heterogeneous disease, both, phenotypically as well as with respect to its molecular biologically. It is, therefore, extremely difficult to establish a diagnostically and prognostically relevant tumourigenesis model. Emerging new techniques such as microarrays, will provide us with a wealth of additional data over the next years. The precise sampling of tumour material in clearly defined histopathological lesions will be a prerequisite for the assignment of specific genetic alterations to defined stages of breast disease.

\section{Introduction}

Breast cancer represents a significant worldwide public health problem [29]. Although considerable progress has been made in searching for the genetic events that underlie the progression of many malignancies $[13,18,44]$, those involved in breast cancer development and progression are still poorly understood [4, 25]. Several molecular genetic analysis have identified alterations in breast tumours that are probably involved in carcinogenesis $[5,26]$. However, the chronology of

\footnotetext{
*Correspondence to: Dr Michaela Aubele, GSF - Forschungszentrum, Institut für Pathologie, Ingolstädter Landstraße 1, 85764 Neuherberg, Germany. Tel.: +49 893187 4132; Fax: +49 893187 3360; E-mail: aubele@gsf.de.
}

these events has not been established. Furthermore, the relationship between histological progression and genetic events in breast cancer is not yet well defined. This review discusses the cytogenetic and molecular genetic findings published within the last years, the problem of correlating molecular-biological data with histology, and, consequently, the difficulties in developing a model for breast cancer progression.

\section{Evidences for intratumoural heterogeneity}

Intratumour phenotypic heterogeneity is one of the characteristics of breast carcinoma, and genetic mechanisms are likely to contribute to it [39]. Heterogeneous molecular findings in different parts of a tumour may reflect concomitant or successive clonal development. No studies have yet been carried out to make a comprehensive analysis of known genetic alterations. The latter, however, would be essential to obtain better insight into the complex system of interactions in the multistep carcinogenesis of breast cancer.

\subsection{Morphological aspects}

Invasive breast carcinomas represent a heterogeneous group of different histopathological entities comprising invasive ductal carcinoma (not otherwise specified - NOS), invasive lobular carcinoma and specific types of breast cancer [36]. In the specific types, e.g., tubular, medullary and mucinous breast carcinoma, the distinct morphological features should amount to at least $75 \%$ of the tumour area to decide for the diagnosis. Therefore, the invasive parts of these tumours are in a histological sense rather homogenous. Invasive lobular carcinomas can, besides the 'classical' linear growth pattern (indian files), display substantial elements of solid, tubulolobular and alveolar variants [36]. A variable histological pattern is also of- 
ten found in the invasive ductal carcinoma NOS, which represent the majority of breast cancer. These tumours may exhibit microscopic foci of solid, tubular, cribriform, medullary, mucinous or papillary differentiation. Histological grading of invasive ductal carcinoma NOS takes into consideration cytological features of differentiation as well as the growth pattern of the invasive tumour, i.e., nuclear hyperchromasia, mitotic rate, and the extent of tubule formation [11]. The grading is usually expressed in three categories: well differentiated (grade I), intermediate (grade II), and poorly differentiated (grade III). This approach is only an estimate for cell differentiation and is based upon the examination of multiple tumour areas which may exhibit heterogeneous cytological features or growth patterns. In all histological breast carcinoma types, however, variable amounts of stroma, normal and/or proliferative glands and ducts, or in situ carcinoma contribute widely to an even more heterogeneous image. If not otherwise stated in this review we focus on the heterogeneity of ductal carcinoma.

\subsection{Conventional cytogenetic}

Some of the earliest indications of genetic abnormalities in breast cancer have come from karyotypic studies [26]. Although several hundred breast cancers were completely karyotyped after short-termed culturing, no specific characteristic cytogenetic abnormality has been observed so far [26]. The most common observations were numerical changes (trisomy of chromosome 7 and 18) and complete or partial monosomy of chromosomes $6,8,11,13,16,17,22$, and X [26].

The most surprising discovery in breast cancer cytogenetics has been that cytogenetically unrelated clones were often found [17,31]. Teixeira et al. [43] first reported the localization of karyotypically distinct cell populations to separate intratumourous domains in breast cancer. Comparison between breast carcinomas and their lymph node metastases [32] has shown that such a polyclonality may exist in both the primary and secondary tumour. Multiclonality has also been documented in a small series of extensively studied multifocal carcinomas $[42,43]$. Cytogenetic analysis of in situ carcinoma of the breast has been carried out only in a small number of cases [16,26], and it could be shown that unrelated clones are a feature of both premalignant breast lesions and overt, infiltrating and metastatic carcinomas [17]. Therefore, one cannot draw any conclusions whether this phenomenon is more characteristic for early or late tumourigenesis.
The findings of cytogenetically unrelated clones in many studies supports the conclusion that a substantial proportion of breast tumours may be polyclonal. But whether they reflect true polyclonal tumourigenesis or not, they are unquestionably manifestations of intratumour genotypic heterogeneity. To understand how the various clones interact during mammary carcinogenesis will be a major task in future breast cancer research.

\subsection{FISH}

Fluorescence in situ hybridization (FISH) analysis is a useful technique for investigating genetic abnormalities in interphase nuclei. A wide variety of DNA probes are available today for FISH, including chromosomespecific probes which hybridize to alpha-satellite pericentromeric DNA regions (to detect changes in chromosome number) and locus-specific probes (to detect amplifications, deletions, breakpoints, or rearrangements) $[26,45]$. FISH analysis of archival material has been the subject of several studies of breast tissue and polysomy of chromosomes 1,16 , and 18 were repeatedly found in carcinoma in situ as well as in invasive breast cancer [16,26,28,39]. Using a panel of centromere specific DNA probes for chromosomes 1 , $3,7,8$, and 16 clonal numeric alterations were similarily observed in the primary tumours as well as in metastases [39]. Consequently, the authors concluded that dominant clones within the primary carcinoma become metastatic. In some of the cases investigated by Fiegl et al. [14] using probes for centromere 11 and 17 metastases showed more complex numerical alterations than those seen in the primary tumour, whereas in other cases fewer aberrations were detected in the metastases. Those results would suggest that heterogeneity evolves in primary and metastatic lesions in a similar manner. Although several FISH studies have attempted to identify genetic alterations responsible for breast tumourigenesis and progression no specific chromosomal alteration could yet be attached to certain histopathological stages.

\section{4. $C G H$}

Comparative genomic hybridization $(\mathrm{CGH})$ is a relatively new molecular-cytogenetic assay [22]. In contrast to FISH, CGH allows for an overview of DNA sequence copy numbers in a single hybridization [19]. Several CGH studies with DNA isolated from paraffin sections of breast cancers described a series of aberrations $[19,23,35]$. Their results confirm a complex pat- 
tern of chromosomal imbalances involving many chromosomes with gains of DNA on chromosomal regions $1 \mathrm{q}, 6 \mathrm{p}, 8 \mathrm{q}, 11 \mathrm{q}, 12 \mathrm{q}, 17 \mathrm{q}$, and 20q, and losses on $6 \mathrm{q}$ and 12q. For this approach, however, several serial sections from the tumour tissue are needed and DNA is isolated from entire sections. Thus, intratumourous heterogeneity can hardly be detected $[19,23]$. By applying a combination of laser-microdissection and whole genomic amplification (DOP-PCR) [1] to examine several small tumour cell samples from one tumourous area revealed several consistent chromosomal changes, which correspond mainly to gains or losses described in other studies. However, this strategy revealed intratumoural heterogeneity, with additional chromosomal aberrations found in the different tumour areas analyzed [1]. CGH analysis of ductal carcinoma in situ (DCIS) has also demonstrated a large number of chromosomal alterations including gains of 1q, 6q, 8q, 17q, $19 \mathrm{q}, 20$ and Xq, and losses on 13q, 16q, 17p, and $22 \mathrm{q}$ $[2,6,20]$. These alterations are similar to those identified in invasive carcinoma, adding weight to the idea that DCIS is a precursor lesion. Proliferative breast lesions are regarded as to be associated with increased risk of developing breast cancer [30,41]. Using CGH combined with laser-microdissection it could be shown that some chromosomal aberrations are already present in proliferative diseases, and that further histopathological stages are accompanied by an increasing number of chromsomal imbalances [3]. However, heterogeneity of the proliferative lesions or intraductal neoplasia has not been investigated so far by $\mathrm{CGH}$.

\subsection{Microarrays}

The use of metaphase chromosomes as the hybridization target limites the resolution of CGH to 10$15 \mathrm{Mb}$. Solinas-Toldo et al. [40] and Pinkel and colleagues [33] chose to replace conventional metaphase chromosome targets with a microarray consisting of an ordered set of DNA fragments in the form of either DNA libraries, P1/PAC clones or cosmids. This approach provides a resolution at least a factor one hundred better than standard $\mathrm{CGH}$, as it is determined only by the size and spacing of the target genomic clones $[33,40]$. Those DNA target arrays may be ideal for identification of DNA gains or losses in tumourous DNA at high-resolution, and an automated evaluation may allow genome-wide screening as a diagnostic and/or prognostic tool. Using the array technology for the investigation of gene expression will make it possible to view all expressed genes of an organism and to detect change in expression under given conditions $[21,38]$. For those expression analysis, cDNA-sized fragments or short (25 nucleotide) oligonucleotides are spotted onto slides or a nylon membrane, and tumourous RNA is hybridized onto the target slides [21, 38].

\section{6. $\mathrm{LOH}$}

Since the introduction of microsatellite-based loss of heterozygosity (LOH) methodology by Cavenee [7] there have been a large number of studies investigating allelic imbalance in invasive breast carcinomas at a large number of chromosomal loci [26]. It is clear from the available data that the pattern of $\mathrm{LOH}$ is very complex, involving numerous chromosomal locations. $\mathrm{LOH}$ has also been investigated in preinvasive lesions. O'Conell et al. [9] have carried out studies using a variety of chromosomal markers and have shown that 50 percent of DCIS share their LOH patterns with invasive carcinoma. This provided preliminary molecular genetic evidence that these lesions are likely to be precursors of invasive carcinoma. Munn et al. [27] found that LOH on the same loci on chromosome 1 were not present in all ducts of carcinoma in situ within the same tumour. Koreth et al. [24] found heterogeneity for LOH at chromosome 11q23 in DCIS and invasive carcinoma from the same patient. Clonal microsatellite alterations have also been observed in atypical ductal hyperplasias (ADHs) and their concurrent ductal carcinoma in situ components, supporting the postulated model in which carcinomas may arise from proliferative lesions [34,37]. In contrast, other studies have shown that microsatellite alterations identified in ADHs from a patient were not shared by the concomitant DCIS or invasive carcinoma examined. Fujii et al. [15] discovered, that $40 \%$ of cases with synchronous DCIS and invasive ductal carcinoma contained heterogeneous patterns of allelic loss at one or more loci in the DCIS components.

There is a considerable body of literature about $\mathrm{LOH}$ studies, and it is not possible to cover all data here. Keeping in mind the molecular heterogeneity of breast tumours it is not surprising that the correlation of $\mathrm{LOH}$ findings with specific stages of the disease remains controversal, and that a great variation in $\mathrm{LOH}$ frequencies at the same loci is found in different studies. Application of refined microdissection methods such as laser-based techniques for sampling of 'pure' cell populations may allow us to better assign allelic losses to distinct histopathological stages of breast disease. 


\section{Breast carcinogenesis model}

The hypothetical multistep model for breast carcinogenesis indicates that invasive carcinoma arises via a series of intermediate hyperplastic (with and without atypia) and neoplastic (in situ carcinoma) stages [4, 26]. Genetic aberrations during this process would lead to the loss or activation of a number of critical genes, such as those involved in cell proliferation, differentiation, and apoptosis $[5,13]$. Further tumour progression is currently believed to result from greater genetic instability $[5,8]$. In contrast to this single progressional pathway a parallel progression from morphologically normal epithelium directly to advanced disease is supported by the discovery of many small invasive cancers that do not have accompanying atypical components. For such a progression a kind of 'field effect' could be assumed [29] provided that some of the genetic alterations found in malignant tumours already exist in the morphologically normal-looking breast epithelium [10]. Some molecular observations indicated that breast disease can potentially follow several different tumourigenic pathways resulting in a more complex picture of the disease $[17,29]$. In contrast to other malignancies such as colorectal carcinoma where a well defined preinvasive lesion in the form of adenoma exist [13], studies in the breast have been complicated by the morphological heterogeneity $[26,30]$ as well as the extremely heterogeneous molecular biological findings such as those described above. Thus, an order of genetic events in the development and progression of breast cancer has not yet been established $[4,5]$.

\section{How to overcome the heterogeneity problem?}

\subsection{Methodological limitations}

To overcome the heterogeneity problem it is necessary at first to investigate only histologically or cytologically clearly defined lesions, to separate nonneoplastic from neoplastic cells, and to distinguish between different histopathological stages. Several studies have begun to examine the genotype-phenotype relationship in ductal carcinoma in situ, in invasive carcinoma, and in metastatic progression using a range of methodologies. The majority of those studies, however, have analyzed certain histopathological stages of breast tumour development such as DCIS or invasive carcinoma as homogeneous group. To get more insight into the degree of heterogeneity multiple samples from lesions of the same histopathology within one patient should be separately studied. Only a few studies have reported such an extensive analysis, and their results have delivered the first evidence of cytogenetically unrelated clones present in premalignant, invasive and metastatic disease $[14,42,43]$. In this context, FISH has distinct advantages, because it allows for the detection of small subpopulations of genetically distinct cells, and reveals cellular heterogeneity in direct correlation with the histological pattern. However, FISH is limited by the number of probes that can be hybridized at any one time and it is still unclear which probes are predictive for distinct carcinogenesis stages or can even give prognostic information.

\subsection{Problem of interpretation}

Instead of the more extensive studies discussed above an alternative would be to examine several samples with the same histopathology taken from a single section or to use FISH analysis on several serial sections. Such experiments may deliver only part of the solution, but are practical approaches to solve the problem of heterogeneity. Some important genetic observations have already been made using this approach, and more data will be collected in the near future. Analysis of the interaction between genetic alterations and their interdependence requires complex multivariate statistical analyses to extract relevant information [12]. With such approaches it might also be possible to discount irrelevant genetic alterations (due to random genetic damage) in breast cancer, which has yet to be defined. The complexity of collected data, e.g., by the microarray [12] or CGH techniques and the necessity to identify patterns in interdependent biological processes will than certainly represent new analytical challenges.

\section{Conclusions}

We are not yet able to understand which genetic alterations correlate with the histopathological findings in breast cancer, and it remains difficult to identify critical genes associated with the histological steps of tumour development or progression. The rapid increase in molecular-biological factors under investigation and the introduction and improvement of technologies such as microarrays will provide us with a wealth of additional molecular data. With this new era comes the promise of quantifying all expressed RNAs and de- 
tecting genetic alterations in normal, proliferative and neoplastic breast tissue. Tissue heterogeneity has been described in the literature but many questions remain unanswered. Are the identified molecular events pathogenetically significant? Can genetic alterations be used to differentiate between the different morphological variants of preinvasive lesions and hence be useful diagnostically? Will we successfully identify prognostic factors and thus be able to give essential support for therapy decision? Investigation of histologically clearly defined lesions and a precise sampling of 'pure' cell populations are the main prerequisite for future molecular-biological studies. Furthermore, more extensive studies are needed to extend our knowledge about genetic changes in breast tumours. Finally, we have to be aware that breast tumours are extremely heterogeneous which complicates data interpretation.

\section{References}

[1] M. Aubele, A. Mattis, H. Zitzelsberger, A. Walch, M. Kremer, P. Hutzler, H. Höfler and M. Werner, Intratumoral heterogeneity in breast carcinoma revealed by laser-microdissection and comparative genomic hybridization, Cancer Genet. Cytogenet. 110 (1999), 94-102.

[2] M. Aubele, A. Mattis, H. Zitzelsberger, A. Walch, M. Kremer, G. Welzl, H. Höfler and M. Werner, Extensive ductal carcinoma in situ with small foci of invasive ductal carcinoma: evidence of genetic resemblance by CGH, Int. J. Cancer (1999) in press.

[3] M. Aubele, M. Cummings, A. Mattis, H. Zitzelsberger, A. Walch, M. Kremer, H. Höfler and M. Werner, Accumulation of chromosomal imbalances from intraductal proliferative lesions to adjacent in situ and invasive ductal breast cancer, Submitted to Diagnostic Molecul. Pathol. (1999).

[4] M.W. Beckmann, D. Niederacher, H.G. Schnurch, B.A. Gusterson and H.G. Bender, Multistep carcinogenesis of breast cancer and tumour heterogeneity, J. Mol. Med. 75 (1997), 429-439.

[5] Bieche and R. Lidereau, Genetic alterations in breast cancer, Genes Chromosomes Cancer 14 (1995), 227-251.

[6] H. Buerger, F. Otterbach, R. Simon, C. Poremba, R. Diallo, T. Decker, L. Riethdorf, C. Brinkschmidt, B. DockhornDworniczak and W. Boecker, Comparative genomic hybridization of ductal carcinoma in situ of the breast - evidence of multiple genetic pathways, J. Pathol. 187 (1999), 396-402.

[7] W.K. Cavenee, T.P. Dryja, R.A. Phillips, W.F. Benedict, R. Godbout, B.L. Gallie, A.L. Murphree, L.C. Strong and R.L. White, Expression of recessive alleles by chromosomal mechanisms in retinoblastoma, Nature 305 (1983), 779-784.

[8] J.-A.W. Chapman, E. Wolman, S.R. Wolman, Y. Remvikos, S. Shackney, D.E. Axelrod, H. Baisch, I.J. Christensen, R.A. White, L.S. Liebovitch, D.H. Moore, F.M. Waldman, C.J. Cornelisse and T.V. Shankey, Assessing genetic markers of tumour progression in the context of intratumour heterogeneity, Cytometry 31 (1998), 67-73.
[9] P. O’Connell, V. Pekkel, S. Fuqua, C.K. Osborne and D.C. Allred, Molecular genetic studies of early breast cancer evolution, Breast Cancer Res. Treatm. 32 (1994), 5-12.

[10] G. Deng, Y. Lu, G. Zlotnikov, A.D. Thor and H.S. Smith, Loss of heterozygosity in normal tissue adjacent to breast carcinomas, Science 274 (1996), 2057-2059.

[11] C.W. Elston and J.D. Ellis, Pathological prognostic factors in breast cancer. I. The value of histological grade in breast cancer: experience from a large study with long-term follow-up, Histopathol. 19 (1991), 403-410.

[12] O. Ermolaeva, M. Rastogi, K.D. Pruitt, G.D. Schuler, M.L. Bittner, Y. Chen, R. Simon, P. Meltzer, J.M. Trent and M.S. Boguski, Data management and analysis for gene expression arrays, Nature Genetics 20 (1998), 19-23.

[13] E.R. Fearon and B. Vogelstein, A genetic model for colorectal tumorigenesis, Cell 61 (1990), 759-767.

[14] M. Fiegl, C. Tueni, T. Schenk, R. Jakesz, M. Gnant, A. Reiner, M. Rudas, H. Pirc-Danoewinata, C. Marosi, H. Huber and J. Drach, Interphase cytogenetics reveals a high incidence of aneuploidy and intra-tumour heterogeneity in breast cancer, $\mathrm{Br}$. J. Cancer 72 (1995), 51-55.

[15] H. Fujii, R. Szumel, C. Marsh, W. Zhou and E. Gabrielson, Genetic progression, histological grade, and allelic loss in ductal carcinoma in situ of the breast, Cancer Res. 56 (1996), 52605265.

[16] M. Harrison, H.M. Magee, J. Loughlin, T.F. Gorey and P.A. Dervan, Chromosome 1 aneusomy, identified by interphase cytogenetics, in mammographically detected ductal carcinoma in situ of the breast, J. Pathol. 175 (1995), 303-309.

[17] S. Heim, M.R. Teixeira, C.U. Dietrich and N. Pandis, Cytogenetic polyclonality in tumors of the breast, Cancer Genet. Cytogenet. 95 (1997), 16-19.

[18] K. Heselmeyer, E. Schröck, S. duManoir, H. Blegen, K. Shah, R. Steinbeck, G. Auer and T. Ried, Gain of chromosome 3q defines the transition from severe dysplasia to invasive carcinoma of the uterine cervix, Proc. Natl. Acad. Sci. USA 93 (1996), 479-484.

[19] J.J. Isola, O.-P. Kallioniemi, L.W. Chu, S.A.W. Fuqua, S.G. Hilsenbeck, C.K. Osborne and F.M. Waldman, Genetic aberrations detected by comparative genomic hybidization predict outcome in node-negative breast cancer, Am. J. Pathol. 147 (1995), 905-911.

[20] L.A. James, E.L.D. Mitchell, L. Menasce and J.M. Varley, Comparative genomic hybridization of ductal carcinoma in situ of the breast: identification of regions of DNA amplification and deletion in common with invasive breast carcinoma, Oncogene 14 (1997), 1059-1065.

[21] M. Johnston, Gene chips: array of hope for understanding gene regulation, Current Biol. 8 (1998), R171-R174.

[22] Kallioniemi, O.P. Kallioniemi, D. Sudar, D. Rutovitz, J.W. Gray, F. Waldman and D. Pinkel, Comparative genomic hybridization for molecular cytogenetic analysis of solid tumors, Science 258 (1992), 818-821.

[23] Kallioniemi, O.P. Kallioniemi, J. Piper, M. Tanner, T. Stokke, L. Chen, H.S. Smith, D. Pinkel, J.W. Gray and F.M. Waldman, Detection and mapping of amplified DNA sequences in breast cancer by comparative genomic hybridization, Proc. Natl. Acad. Sci. USA 91 (1994), 2156-2160. 
[24] J. Koreth, P.B. Bethwaite and J.O.D. McGee, Mutation at chromsome 11q23 in human non-familial breast cancer: a microdissection microsatellite analysis, J. Pathol. 176 (1995), 1118.

[25] T. Kuukasjärvi, R. Karhu, M. Tanner, M. Kahkonen, A. Schaffer, N. Nupponen, S. Pennanen, A. Kallioniemi, O.P. Kallioniemi and J. Isola, Genetic heterogeneity and clonal evolution underlying development of asynchronous metastasis in human breast cancer, Cancer Res. 57 (1997), 1597-1604.

[26] S.R. Lakhani, The transition from hyperplasia to invasive carcinoma of the breast, J. Pathol. 187 (1999), 272-278.

[27] K.E. Munn, R.A. Walker and J.M. Varley, Frequent alterations of chromsome 1 in ductal carcinoma in situ of the breast, Oncogene 10 (1995), 1653-1657.

[28] D.S. Murphy, S.F. Hoare, J.J. Going, E.A. Mallon, W.D. George, S.B. Kaye, R. Brown, D.M. Black and W.N. Keith, Characterization of extensive genetic alterations in ductal carcinoma in situ by fluorescence in situ hybridization and molecular analysis, J. Natl. Cancer Inst. 87 (1995), 1694-1704

[29] I.F. Newsham, The long and short of chromosome 11 in breast cancer, Am. J. Pathol. 153 (1998), 5-9.

[30] D.L. Page, W.D. Dupont, L.W. Rogers and M.S. Rados, Atypical hyperplastic lesions of the female breast. A long-term follow-up study, Cancer 55 (1985), 2698-2708.

[31] N. Pandis, Y. Jin, L. Gorunova, C. Petersson, G. Bardi, I. Idvall, B. Johansson, C. Ingvar, N. Mandahl, F. Mitelman and S. Heim, Chromosome analysis of 97 primary breast carcinomas: identification of eight karyotypic subgroups, Genes Chromsomes Cancer 12 (1995), 173-185.

[32] N. Pandis, M.R. Teixeira, A. Adeyinka, H. Rizou, G. Bardi, F. Mertens, J.A. Andersen, L. Bondeson, K. Sfikas, H. Qvist, N. Apostolikas, F. Mitelman and S. Heim, Cytogenetic comparison of primary tumors and lymph node metastases in breast cancer patients, Genes Chromosomes Cancer 22 (1998), 122129.

[33] D. Pinkel, R. Segraves, D. Sudar, S. Clark, I. Poole, D. Kowbel, C. Collins, W.-L. Kuo, C. Chen, Y. Zhai, S.H. Dairkee, B.-M. Ljung, J.W. Gray and D.G. Albertson, High resolution analysis of DNA copy number variation using comparative genomic hybridization to microarrays, Nature Genetics $\mathbf{2 0}$ (1998), 207-211.

[34] D.M. Radford, K.L. Fair, N.J. Phillips, J.H. Ritter, T. Steinbrueck, M.S. Holt and H. Donis-Keller, Allelotyping of ductal carcinoma in situ of the breast: deletion of loci on 8p, 13q, 16q, 17p and 17q, Cancer Res. 55 (1995), 3399-3405.

[35] T. Ried, K.E. Just, H. Holtgreve-Grez, S. duManoir, M.R. Speicher, E. Schröck, C. Latham, H. Blegen, A. Zetterberg, T. Cremer and G. Auer, Comparative genomic hybridization of formalin-fixed, paraffin-embedded breast tumors revealed different patterns of chromosomal gains and losses in fibroadenomas and diploid and aneuploid carcinomas, Cancer Res. 15 (1995), 5415-5420.

[36] P.P. Rosen, H.A. Oberman and AFIP (Armed Forces Institute of Pathology), Tumors of the Mammary Gland, 3rd edn, Washington DC, 1993.

[37] C.L. Rosenberg, P.S. Larson, J.D. Romo, A. de las Morenas and D.V. Faller, Microsatellite alterations indicating monoclonality in atypical hyperplasias associated with breast cancer, Hum. Pathol. 28 (1997), 214-219.

[38] M. Schena, D. Shalon, R.W. Davis and P.O. Brown, Quantitative monitoring of gene expression patterns with a complementary DNA microarray, Science 270 (1995), 467-470.

[39] J.F. Simpson, D.E. Quan, J.P. Ho and M.L. Slowak, Genetic heterogeneity of primary and metastatic breast carcinoma defined by fluorescence in situ hybridization, Am. J. Pathol. 149 (1996), 751-758.

[40] S. Solinas-Toldo, S. Lampel, S. Stilgenbauer, J. Nickolenko, A. Benner, H. Dohner, T. Cremer and P. Lichter, Matrix-based comparative genomic hybridization: biochips to screen for genomic imbalances, Genes Chromosomes Cancer 20 (1997), 399-407.

[41] F.A. Tavassoli and H.J. Norris, A comparison of the results of long-term follow-up for atypical intraductal hyperplasia and intraductal hyperplasia of the breast, Cancer 65 (1990), 518-529.

[42] M.R. Teixeira, N. Pandis, G. Bardi, J.A. Andersen, N. Mandahl, F. Mitelman and S. Heim, Cytogenetic analysis of multifocal breast carcinomas: detection of karyotypically unrelated clones as well as clonal similarities between tumor foci, $\mathrm{Br}$. $\mathrm{J}$. Cancer 70 (1994), 922-927.

[43] M.R. Teixeira, N. Pandis, G. Bardi, J.A. Andersen, F. Mitelman and S. Heim, Clonal heterogeneity in breast cancer: karyotypic comparisons of multiple intra- and extra-tumorous samples from 3 patients, Int. J. Cancer 63 (1995), 63-68.

[44] R.G. Weber, J. Bostrom, M. Wolter, M. Baudis, V.P. Collins, G. Reifenberger and P. Lichter, Analysis of genomic alterations in benign, atypical, and anaplastic meningiomas: toward a genetic model of meningioma progression, Proc. Natl. Acad. Sci. USA 94 (1997), 14 719-14 724.

[45] M. Werner, L. Wilkens, M. Aubele, M. Nolte, H. Zitzelsberger and P. Komminoth, Interphase cytogenetics in pathology: principles, methods, and applications of fluorescence in situ hybridization (FISH), Histochem. Cell. Biol. 108 (1997), 381390 . 


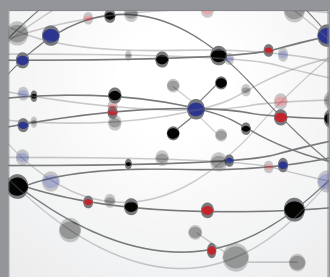

The Scientific World Journal
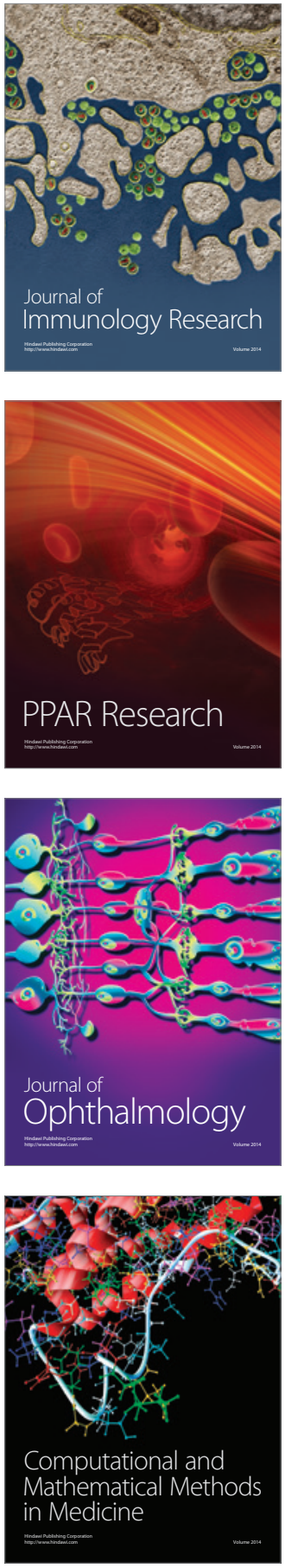

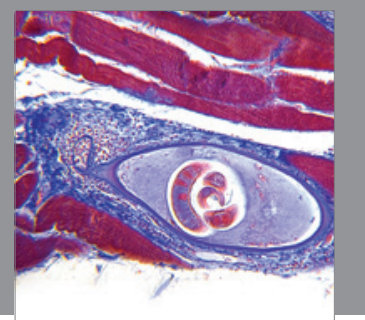

Gastroenterology

Research and Practice
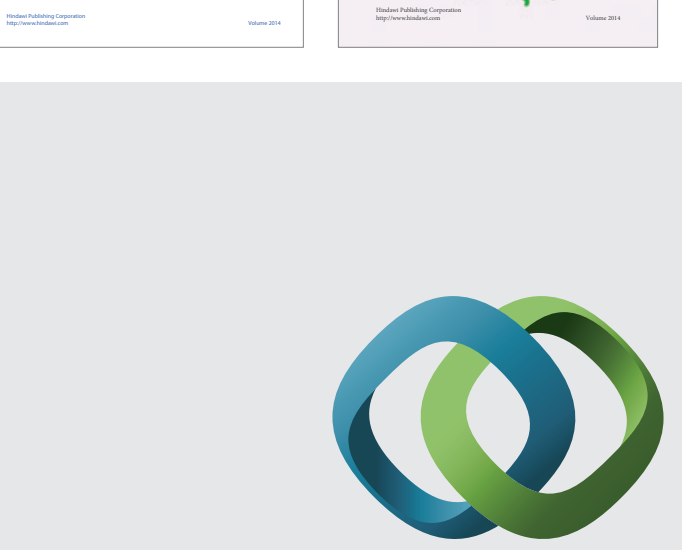

\section{Hindawi}

Submit your manuscripts at

http://www.hindawi.com
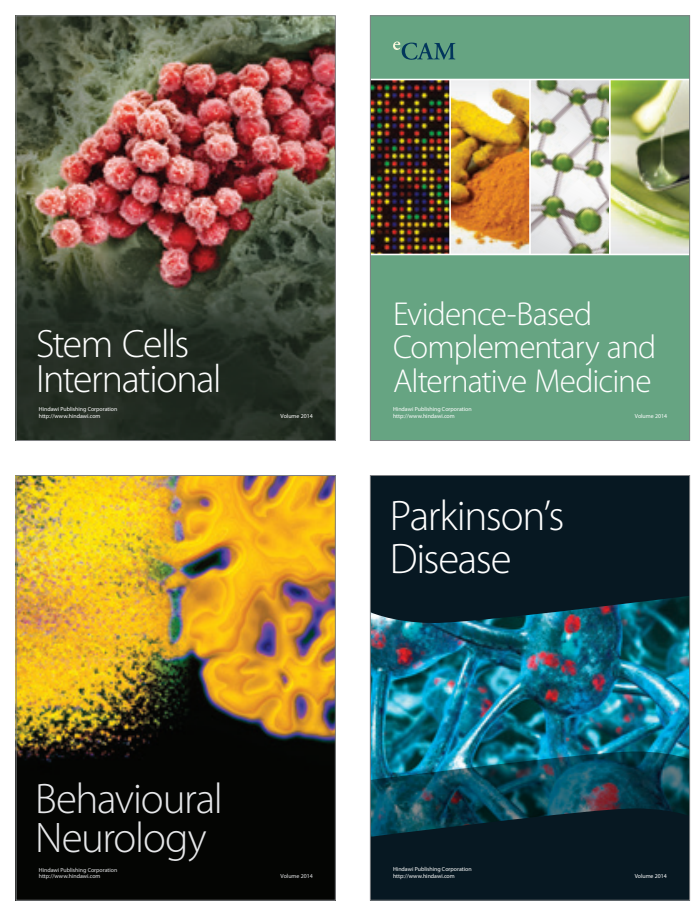

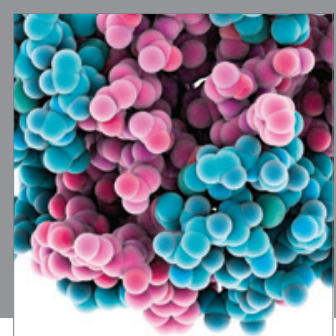

Journal of
Diabetes Research

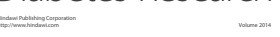

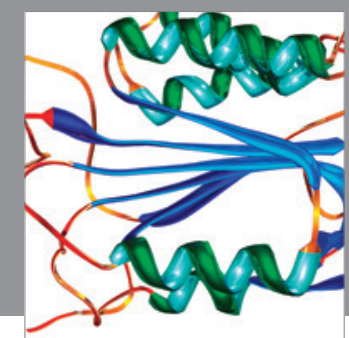

Disease Markers
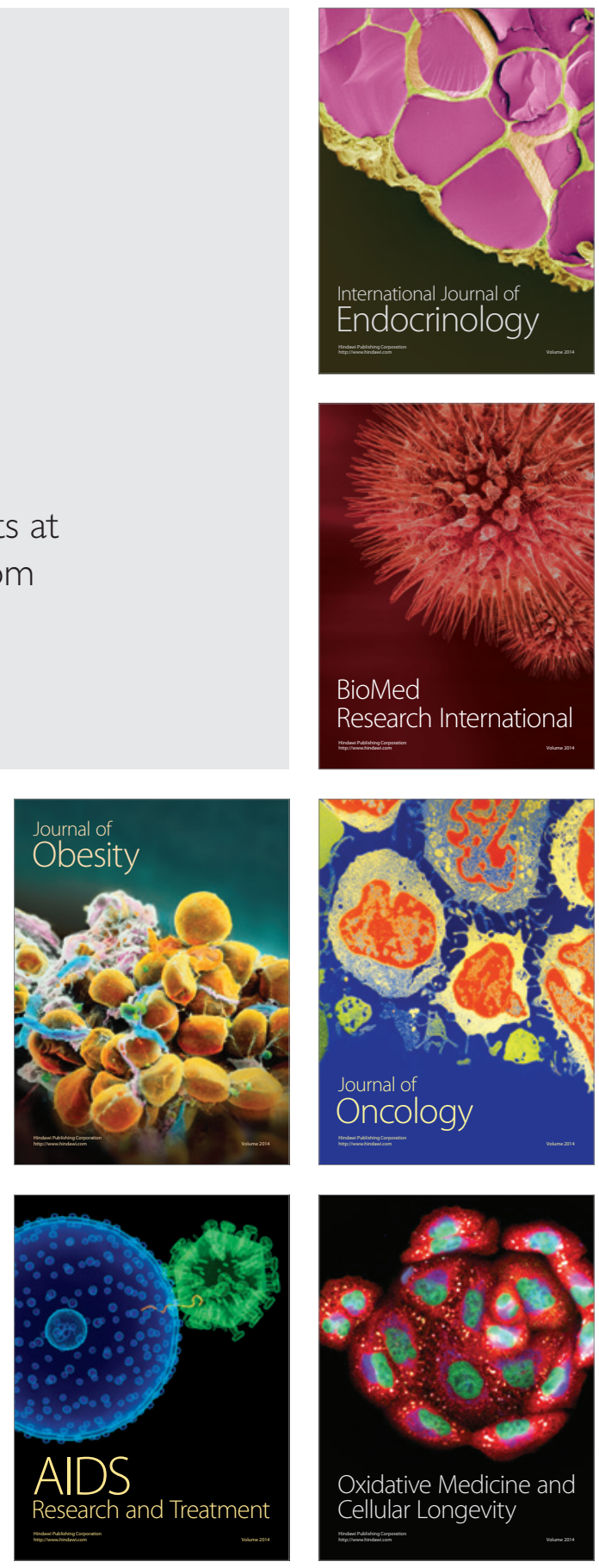